\title{
Application Research of AR (Augmented Reality) Technology in Creative Interaction of Poster Design
}

\author{
Wenqian Deng ${ }^{1, *}$ Yunxiu $\mathrm{Wu}^{1}$
}

\author{
${ }^{1}$ College of Art and Design, Wuhan Textile University, Wuhan, Hubei, China \\ *Corresponding author. Email: 670138792@qq.com
}

\begin{abstract}
Through the application of AR technology in the creative interaction of poster design, this paper is carried out from the aspects of public welfare poster, commercial poster, cultural poster and social poster in the poster design. It studies the design expression forms generated by creative interaction of AR technology, and further explores and analyzes the development prospect and influence of augmented reality technology in the interaction of poster design, from which some problems need consideration in depth are found out. In the modern society with advanced science and technology, the interaction of AR technology in poster design develops rapidly, which has a rapid impact on the whole graphic design. However, will this design form change the original artistic nature of poster design? It is important for poster design to retain its own charms in the rapid development of science and technology and not be submerged by the trend of intelligent technology, so that the audience will keep their interest in, appreciate and guess the real meaning and information of the design work itself while interacting with the design work.
\end{abstract}

Keywords: AR technology, poster design, transitional information, versatility, art

\section{INTRODUCTION}

AR is short for Augmented Reality. It is a kind of virtual element made by computer synthesis. It is developed through VR technology (virtual reality technology), which seamlessly connects the virtual and the real. After the simulation of images, texts and other virtual information, it is applied to the real world to "augment" the real world. In this way, it brings new visual and real interactive experience to people. Through the rapid development of science and technology, the improvement of software technology and hardware facilities technology enables the rapid growth of AR technology, which is then adopted to various industries. It can assist the design and development of virtual interactive applications to show the real space and objects in the field of vision. This unique technical feature has inspired many designers to apply it in their design works. This technology has been around since 1990. It is a smart technology that has been developing for more than 20 years. From the earliest conceptualization to its gradual access to the various fields of life now, it has become increasingly mature and developed into a high-tech intelligent technology product. AR technology is undoubtedly a key to open the door to the new world, as well as a new world landed on by graphic designers. Such technology, when combined with modern poster design, can produce many interesting creative forms. However, such combination has both advantages and disadvantages. When stepping into this new and unknown field, people will also encounter some issues worthy of thinking and exploring.

\section{AN OVERVIEW OF THE POSTER DESIGN}

The poster design occupies the main position in the graphic design. With the strong visual art effect, the functions of propaganda and information dissemination, it is widely used in business, public welfare, cultural communication, social information communication and other fields. It is a creative design activity and process of graphic art to convey information through the combination of graphic, color, text, printmaking and other elements to produce visual impact and other effects. The traditional poster design is a combination of flat graphics, text, etc., which is a two-dimensional display, having the characteristics of large size, strong far-sightedness and high artistic quality. Traditional posters are often put in the most eye-catching places, so they are also called "stickers", giving people a feeling of being clear at a glance. Poster design is divided into several categories: "social posters, public posters, cultural posters, commercial posters and so on. With the rapid development of science and technology, social economy and the constantly improving curiosity of people to receive new things, as the most important poster design in graphic design, it begins to explore 
more creative and artistic possibilities instead of sticking to the traditional two-dimensional plane.

\section{THE PROSPECT OF AR TECHNOLOGY UNDER CREATIVE INTERACTION OF POSTER DESIGN}

AR technology originated in 1990. In 1992, the term was named by Tom Caudell and his colleagues at Boeing Company, and AR was officially born. It is a kind of intelligent technology that can transform the infinite augmentation of reality, realize the natural interaction in the real environment, and achieve the extraordinary sensory experience into 3D. Its emergence has not only played a chemical reaction in the design industry. It has driven the innovation and development of the whole society in various fields, and formed a huge breakthrough and transformation in all walks of life. Sutherland, the father of computer graphics and the father of augmented reality, developed the first set of augmented reality system, which was the first AR device realized by human beings, and was named The Sword of Damocles. AR was formally defined in 1997. People are going to use augmented reality (AR) technology everywhere to enhance their perception and help them see, hear, feel and enrich their environment in new ways. AR will support people in education, maintenance, design and reconnaissance. ${ }^{1}$ Although AR technology was born earlier, its combination with poster design is still in the development stage at present. The birth of AR technology does bring a lot of possibilities to poster design.

Compared with the formalized effect brought by AR technology, the traditional poster design is relatively single. The posters in the streets and even in many design exhibitions will make people feel a little aesthetic fatigue. The poster design has indeed encountered a bottleneck period. The breakthrough of traditional two-dimensional poster design made by AR technology has brought different new visual experience to both designers and the public. The augmented reality function of AR technology can make the twodimensional poster more realistic and interesting to the public. Xu Li and Zou Qin mentioned in "Application and development of augmented reality technology in the field of art design that "The rapid progress of AR technology turns the evolution of posters from static to dynamic. Dynamic posters led to new graphic designs. They convey more abundant graphic information, gives the audience different sensory experience, and enhances the interest and interactivity, which makes a breakthrough in the sense of space, tactile, auditory display of a new three-dimensional poster. The AR

\footnotetext{
Angelia. Do you know the 50 years development history of AR augmented reality technology? [OL]. Digital display online,
} 2018. technology superimposes the real and virtual images into the same picture in real time, forming the effect of "combining the real and the virtual, and interacting in real time", so that the audience can get a sensory experience beyond the reality. ${ }^{2}$ It can be predicted that the development prospects of AR technology application in the poster design is very considerable.

\section{THE EMBODIMENT OF AR TECHNOLOGY UNDER THE CREATIVE INTERACTION OF POSTER DESIGN}

There are many types of poster design, such as film posters, social posters, cultural posters and so on. Although the AR technology was born early, its real application in poster design didn't develop until in recent years, and it quickly set off a boom in the design field. From this point of view, AR technology will be more applied to poster design in the future, and even become the mainstream. However, personally the author holds that it can only be regarded as a unique form of expression in poster design, and it won't replace the traditional colors of poster design.

\section{A. Movie poster}

A movie poster is a poster advertisement designed to achieve better publicity effect before a movie is shown. With the rapid development of movies, the expression forms of movie posters are also being enriched rapidly. The film poster based on the original traditional two-dimensional plane has a very beautiful picture and a unique technique of expression. Moreover, the cultural connotation and commercialization of the film itself make many movie posters worth appreciating and collecting. As a derivative of the film, it was the first stepping-stone to determine the box office. With the addition of AR technology, the propaganda of movies will be more powerful, and the poster may even replace the existence of trailers, since it can provide more sensory experience for audiences. The first company to add AR to the design of movie posters is Facebook of the United States, which is developing an augmented reality experience called "target tracking," allowing users to launch an augmented reality experience by pointing their phone at an image. The poster designs for "Ready Player One" and "A Wrinkle in Time" provide a simple animation experience, which can also be obtained through a quick scan of the QR code ("Fig. 1"). In China's domestic film posters, "The Wolf Totem" also applied AR technology in the film posters. One can experience AR by scanning the QR code on the mobile phone, and can have a real close contact with wolves, as shown in "Fig. 2".

\footnotetext{
Guo Ji Ri Bao, issue 2, 2010.
} 


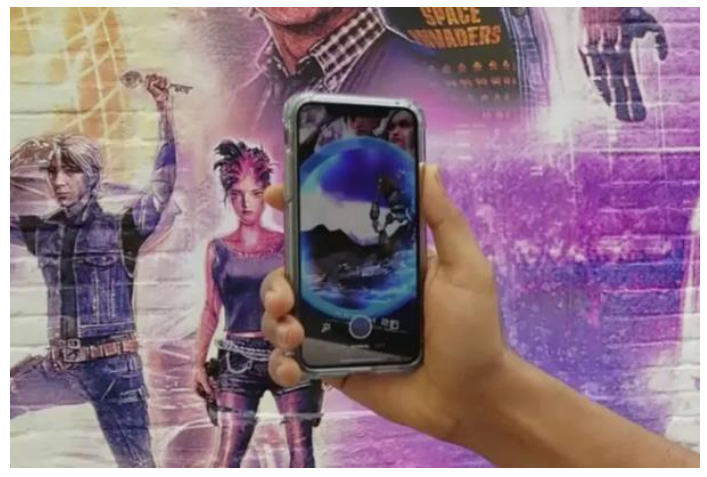

Fig. 1. The poster design for "Ready Player One".

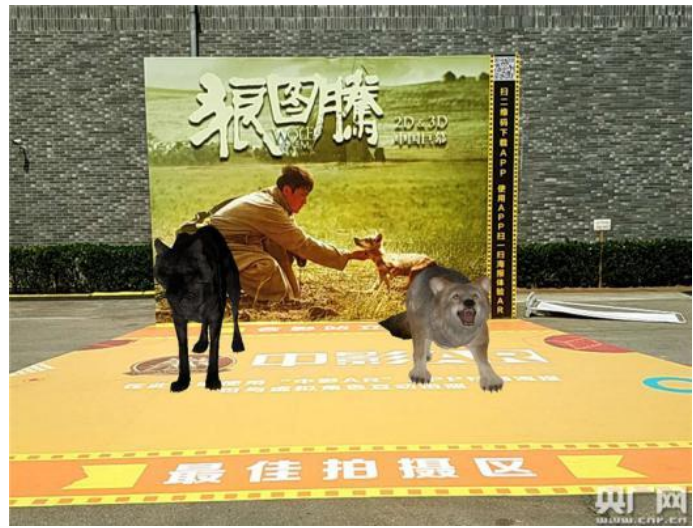

Fig. 2. The poster design for "The Wolf Totem".

The creative interaction of AR technology in movie posters has indeed brought different sensory and visual experiences to the public, and the advance notice of movies is also more three-dimensional and intuitive. This really conforms to the "easy-to-see" purpose of the poster design, and also brings more benefits to the film. This form of expression has already begun to become

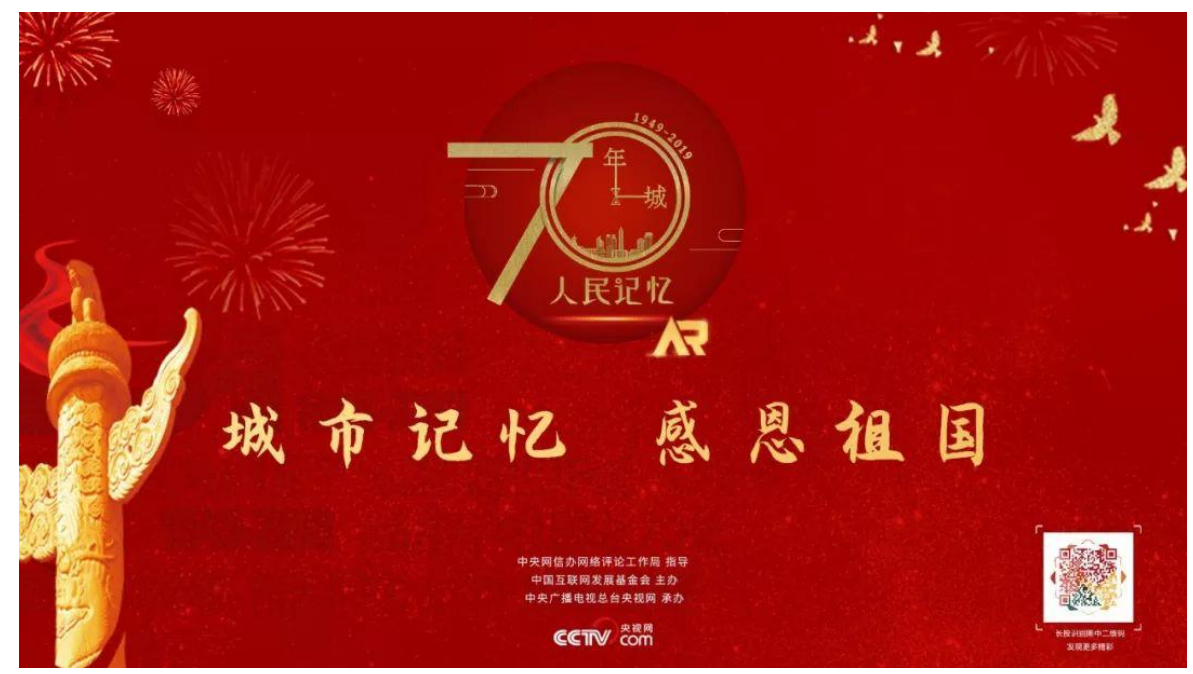

Fig. 3. People's memory: "70 years and 70 cities". the development trend of film poster design in the future. However, if the overwhelming preference is given to one form of artistic expression, will it affect the versatility of poster design?

\section{B. Cultural poster}

Cultural posters are mainly used to publicize various cultural activities and exhibitions, such as culture and exhibition features. In recent years, AR technology has also been applied to cultural posters. Most notably, in 2019, CCTV.com launched the AR interactive poster "people's memory: 70 years and 70 cities" in combination with AR technology and posters, watching a series of short videos of cities to arouse "city memory" and "gratitude for the motherland", as shown in "Fig. 3". The emergence of AR technology elevates the traditional two-dimensional poster to threedimension, which is really eye-catching for the publicity novelty and has a wider range of information. But it lacks artistry and versatility. When more media forms are combined with posters, the public pays more attention to the sense of experience after mobile phone scanning and the form feature of "two birds with one stone", but seems to ignore the posters themselves. Compared with two-dimensional traditional posters, the continuous improvement of science and technology as well as the continuous improvement of all walks of life and fields really need more novel and unique blood for the increasingly improved social trend. However, this is a kind of promotion and increase of uniqueness on the original poster design image, rather than the enthusiastic pursuit and unification of the one-sided views, as the intuitive information expression and artistry present the original intention and lasting appeal of the poster design. 


\section{Creative poster}

Creative posters are often defined in a novel and innovative form according to the creative inspiration and expression of the designer's own feelings. To transmit information appropriately through creative thinking, organization and techniques requires rich esthetic feeling and fresh visual experience. It can come from commercial posters, public service posters and cultural posters. In a word, as long as the expression form of the poster is novel enough, it can be called "creative poster". Creative posters focus on the word "creative," but that's where the difficulty lies. In the past, it was not easy for designers to display and publicize on the two-dimensional posters and achieve the effect of novel and unique information display. It is not as simple as commercial posters to show product information and have a certain scope of definition. Creative posters require designers to brainstorm infinitely and accurately to show information in a unique and visually striking way. AR technology is indeed a unique form of expression for creative posters, which well interprets the word "creativity". ("Fig. 4")
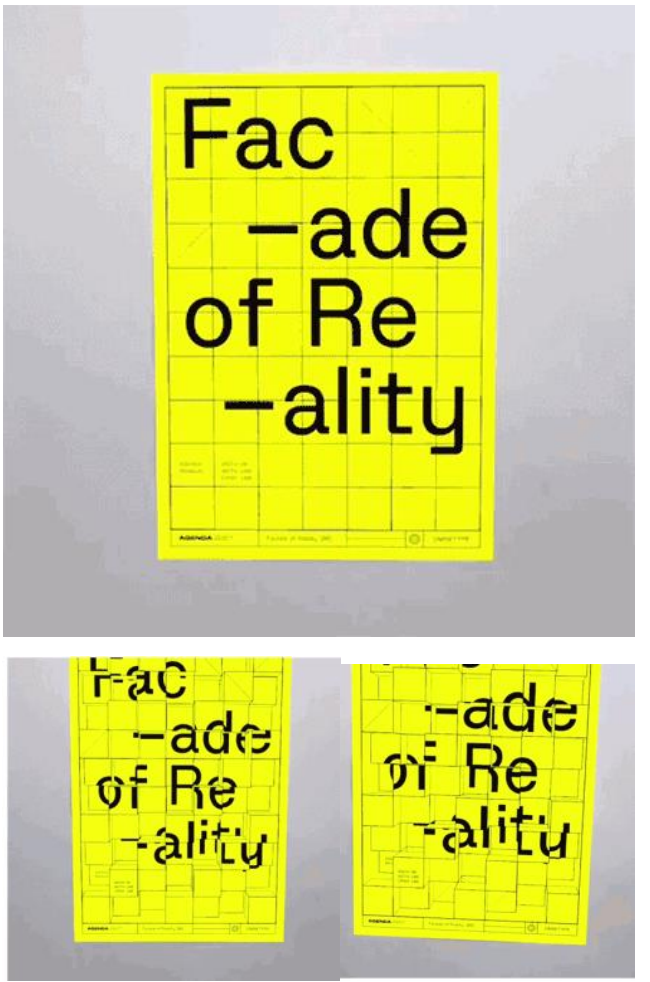

Fig. 4. An AR poster for design exhibition.

\section{THE INFLUENCE OF AR TECHNOLOGY ON THE CREATIVE INTERACTION OF POSTER DESIGN}

The interactive combination of poster design and intelligent science and technology changes the inherent expression form of poster design. The traditional poster planarization is completely transformed into a threedimensional dynamic model, so as to produce another new communication medium for the poster to convey information. The emergence of AR technology makes the poster design more interactive, interesting and experiential with the audience. Through the creative interaction between the augmented reality technology and the graphic design works, the audience can communicate from the perspective of hearing, vision and feeling, which cannot be achieved in the traditional two-dimensional poster. The previous two-dimensional model has some limitations for designers in creation. AR technology has broken through such limitations, but it is still in the stage of continuous development and improvement, with certain instability. In order to better apply it to the poster design, designers still need to rationalize the use of AR technology. They should avoid simplification and overwhelming bias and it is necessary to combine the traditional poster design with the emerging forms and retain the artistic and stylistic features of the traditional poster design.

\section{CONCLUSION}

In conclusion, the application and research of $\mathrm{AR}$ technology and poster design under creative interaction that, AR technology has realized the combination of virtual and real, real-time interaction of new forms of expression. It makes good use of intelligent science and technology in the design so that viewers can have a new experience and expression in terms of visual sense and design expression. This makes the experience more interesting, experimental and real. These cannot be reflected in the previous poster design. But it is also a double-edged sword. On the one hand, it really breaks through the inherent pattern of previous design for designers, and endows poster design with a new way of expression. On the other hand, it is still necessary to define the trend orientation of such a new form. It is not right to blindly or excessively focus on the simplicity and interest in the experience, but ignore the original intention of poster design itself and the artistic value and visual impact of traditional poster design. Only a reasonable combination of the two can produce a better poster design. The plane is not only the plane. More importantly, it keeps the audience focused on the real meaning expressed by the poster design itself.

\section{References}

[1] Zhu Sihe, Wu Fengnan. Application and development of augmented reality technology in the field of art design [J]. Humanities and Technology, 131. (in Chinese)

[2] $\mathrm{Xu} \mathrm{Li}$, Zou Qin. Practical research on poster design innovation under AR technology [J]. Packaging Engineering, 2019, (0909), 22-23. (in Chinese) 
[3] Ma Zenghui. Research on user experience under augmented reality technology [J]. Art \& Technology, 2015, (07). (in Chinese)

[4] Guo Yucheng, Liu Miao. Innovation of image representation in poster design [J]. Packaging Engineering, 2014, (3524), 76-80. (in Chinese)

[5] Dong Li. dynamic poster design practice based on new media [D]. Hubei University of Technology, 2016. (in Chinese)

[6] Liu Xiao. Research on dynamic poster design of Chinese traditional festival theme for new media [D]. Shaanxi University of Science and Technology, 2019. (in Chinese)

[7] Nick Sousanis. Trans. by Yan Anrou. Non-print [M].Beijing United Publishing Company, 2018, (11).

[8] Hu Xiaochen, Deng Bing. Research on the application of augmented reality aided design in virtual interaction [J]. Source of Creative Design, 2015, (02). (in Chinese)

[9] Li Lin, Research on dynamic poster layout design in new media environment [D]. North China University of Science and Technology, 2019. (in Chinese)

[10] Sun Xing, Jin Xin, Zhang Xiaokun, Li Xiaodong, Sun Jianshu. Design and Implementation of Mobile Terminal Augmented Reality System [J]. Journal of Beijing Institute of Electronic Science and Technology, 2017, 12. (in Chinese)

[11] Helen Papagiannis, Ran Xiao, Xiaolei Wang Trans. Augmented human: how does technology shape new realities[M]. Mechanical industry press.

[12] Tristan C. Endsley, Kelly A. Sprehn, etc. AUGMENTED REALITY DESIGN HEURISTICS: DESIGNING FOR DYNAMIC INTERACTIONS [D]. Foreign meeting, 2017.

[13] Irshad, S.; Rambli, D.R.A. User Experience Satisfaction of Mobile-Based AR Advertising Applications [C]. Advances in Visual Informatics. 4th International Visual Informatics Conference, IVIC 2015.

[14] James Young, Mary Barna Bridgeman, EvelynR. HermesDeSantis, Presentation of scientific poster information: Lessons learned from evaluating the impact of content arrangement and use of infographics Currents in Pharmacy Teaching and Learning Volume [J]. 2019, 204-210. 